\title{
Evaluation of the role of Shodhana (Purification) process in Croton tiglium seeds for reduction of toxic content
}

\author{
Research Article
}

\section{Ajay Kumar Meena ${ }^{1 *}$, Poorna Venkataraman², Ravindra Singh ${ }^{3}$, Kusuma Ganji², Murali Krishna $\mathrm{C}^{4}$ and Srikanth $\mathrm{N}^{3}$}

\author{
1. Regional Ayurveda Research Institute, Aamkho, Gwalior, \\ 2. Captain Srinivasa Murthy Central Ayurveda Research Institute, Chennai, \\ 3. Central Council for Research in Ayurvedic Sciences, Ministry of AYUSH, Government of India, New Delhi, \\ 4. Regional Ayurveda Research Institute, Payakapuram, Vijaywada.
}

\begin{abstract}
In Ayurvedic texts, Jayapala or croton tiglium seeds are well known as khumbini for its toxicity (severe purgative action) and are used for the treatment of constipation after shodhana (detoxification) of the seed with godugdha (cow milk). The oil content of the seeds was responsible for the purgative property, and its reduction enhances its medicinal usage. The presence of Crotonoside or iso-guanosine in seed extract was identified in HPTLC and quantified using high pressure liquid chromatography technique both before and after the purification process of the seed. The phytochemicals and physicochemical parameters of seeds were analyzed to find the level of changes in the processed seeds. The other chemical constituents of the seed extracts are studied using different techniques like HPTLC, HPLC, LC-MS, and GC-MS. The study has revealed that the detoxification process, as per classical texts, has shown an effective depletion in the quantity of crotonoside in processed seeds.
\end{abstract}

Key Words: Ayurveda, Purification, Crotonoside, LC-MS, GC-MS.

\section{Introduction}

Croton tiglium L. (Euphorbiaceae) is an important medicinal plant which is commonly known as Jayapala, or Croton oil plant. It is an erect, evergreen shrub/small tree growing up to 7 meters tall and has been grown for these uses for beyond 2,000 years. It is still often cultivated nowadays and also sometimes grown as an ornamental. The roots, seeds, and seed oil are traded within India \& South-East Asia, and the oil is exported to Europe. (1-2)

The plant has a very long tradition of herbal use, being employed as a powerful laxative and oil to treat a wide range of skin problems. It is used to treat constipation, dyspepsia, dysentery, gastrointestinal disorders, intestinal inflammation, rheumatism, peptic ulcer, visceral pain, and headache. (3-5)

All parts of the plant are poisonous. The seeds are recognized to be very poisonous, and are used as a fish poison and criminal activities, four seeds can be a lethal dose for a human adult. Symptoms of croton oil poisoning are at pain starts at the back of the throat and then in the anal canal. A dose of bismuth is know as an immediate antidote. Extreme caution must be taken

* Corresponding Author:

Ajay Kumar Meena

Regional Ayurveda Research Institute,

Aamkho,

Gwalior - 474009 .

India

Email Id: ajaysheera@gmail.com with all medicinal applications of this plant, given its extreme toxicity. (6-9)

Particularly the seeds have been chemically analysed only. Major constituent is a fixed oil named croton oil (30 - 45\%) and protein (20\% approx). The oil comprises the fatty acids - myristic acid, oleic acid, linoleic acid, arachidic acid, palmitic acid, formic acid, stearic acid, acetic acid, and smaller amounts of tiglic acid, butyric acid, lauric acid, and valeric acid. Severe inflammation on the skin is caused by Croton oil. The toxic principles are a group of proteins called 'crotin', about $3.5 \%$ croton resin ('crotonol'), a glucoside called crotonoside (iso-guanosine), and a non-volatile unsaturated fatty acid responsible for the purgative properties. The mixture of toxic proteins croton globulin and croton albumin Crotin. (9)

The plant is known as Kumbhin̄ in Ayurvedic texts and is used to treat constipation after Śhodhana (detoxification process) with Godugdha (cow milk) of the seeds. (10-11)

In the present study, Croton tiglium seeds were purified with cow milk as reported in Ayurvedic classics. The phytochemical constituents of the seed are analyzed to identify the biologically active compounds. The standard Crotonoside (Sigma Aldrich) was purchased and identified using the HPTLC technique and quantified using the HPLC technique for raw and purified seeds and other physicochemical parameters. GC-MS and LC-MS are also performed to extract the seeds to establish the significant reduction of Crotonoside and other fatty acids responsible for the purgative nature after purification. 


\section{Materials and Methods}

\section{Sample collection}

The Jayapala (Croton tiglium Linn) seeds were procured from the local crude drug market Chennai, Tamilnadu, and authenticated at Botany Department of Captain Srinivasa Murthy Regional Ayurveda Drug Development Institute, Chennai, with the help of flora. A voucher specimen was submitted in the department of Botany of the Institute.

\section{Purification/Detoxification}

$500 \mathrm{~g}$ of the dry raw Jayapala seeds are cleaned, weighed, and taken. The outer coat of the testa of the sample was removed with the help of iron mortar and pestle. It is then tied in a muslin cloth and hanged inside the mud pot without touching the bottom. Swedana is performed by dola-yantra in cow's milk for three hours. Cooled, removed and washed with warm water and removed the cotyledon of the seeds with the help of the knife. Dry, powder, and then soak the sample in lemon juice (nimbu swarasa) for three days. Fresh juice was added every day. Then the paste was applied to the outside of the mud pot and dried in sunlight to remove the excess oil content of the seed. Once dried, the powder is collected and used for analysis. (12)

\section{Standardisation of Jayapala seeds}

To ascertain the quality of the procured Jayapala seeds used in the study physico-chemical analysis was done. Qualitative analysis of phytochemicals like alkaloids, tannins, flavonoids, steroids, saponins, phenols, coumarins, glycosides, acids, proteins were analysed. Physico-chemical parameters like Alcohol and Water-soluble extractive values, $\mathrm{pH}$, Total Ash, Acid- insoluble Ash, and Loss on Drying were quantitatively analyzed based on the standard procedures in Ayurvedic pharmacopeia of India (API) methods. (13-15, 9)

\section{Sample/Extract preparation}

$10 \mathrm{~g}$ of both raw and purified samples are taken in thimble along with $250 \mathrm{ml}$ of ethanol in Round Bottom flask. Soxhlet extraction was performed for 6-8 hours, and the extracts are collected. The same procedure was repeated with chloroform and hexane; the extracts are organized and labeled properly. Known quantities of these extracts are taken to prepare the test solution for HPTLC, HPLC, GC-MS, and LCMS analysis.

\section{Reference standard preparation}

Crotonoside or iso-guanosine was taken as the reference standard and was quantified in HPLC. The standard was purchased from Sigma Aldrich, and HPLC grade water was used. $260 \mathrm{ppm}$ of Crotonoside standard was prepared by dissolving in methanol and used for analysis. All other chemicals \& solvents used were of AR grade.

\section{Identification of Bio-marker by HPTLC:}

The residues obtained from ethanol and chloroform extracts were weighed and dissolved in methanol. $2.6 \mathrm{mg}$ of the standard was weighed and prepared in a $10 \mathrm{ml}$ volumetric flask. It was then filtered through $0.22 \mu$ membrane filters and used to identify reference standard Crotonoside - biomarker compound. 151 of each test solution of chloroform, ethanol extract, and Crotonoside reference standard solution were spotted in different tracks on a precoated silica gel 60 $\mathrm{F}_{254}$ TLC plate (E. Merck) of $0.2 \mathrm{~mm}$ thickness. The plate was developed in the suitable solvent system of $N$ butanol: Methanol: Water: Ammonia Solution (25\%) (4:4:1:0.5) and rises to a distance of $8 \mathrm{~cm}$. The plate was observed through CAMAG TLC Visualizer under $\mathrm{UV}$ at $254 \mathrm{~nm}$ and $366 \mathrm{~nm}$, and photos were documented. Finally, the plate was dipped in vanillinsulphuric acid reagent and heated in a hot air oven at $105^{\circ} \mathrm{C}$ until the color of the spots appeared, and the photo was documentation under white light.

\section{Chemical profiling of Croton tiglium seeds}

The chromatographic profiling was performed using four different chromatographic techniques to study the effect of the shodhana process on the Croton tiglium seeds.

\section{HPTLC chromatographic profiling of Croton tiglium seeds}

The Fingerprint profile was performed for the above-developed plate (Figure 1) used for identification. Before derivatization, the plate was scanned under UV at $254 \mathrm{~nm}$ and $366 \mathrm{~nm}$ using deuterium and mercury lamp, respectively. After derivatization plate was scanned at $540 \mathrm{~nm}$ using a tungsten lamp.

\section{GC-MS chromatographic profiling of Croton tiglium seeds}

The test solutions were prepared by dissolving the dried extracts of hexane and chloroform in chloroform and methanol solvents of the desired volume. It is filtered and sent for GC-MS analysis. Here interpretation on mass spectrum GC-MS was conducted by using the database of the National Standard and Technology (NIST) library. The spectrum of the unknown component was compared with the spectrum of the known components stored in the NIST library. The hexane and chloroform extracts of the Croton tiglium seeds before and after the shodhana process, the processed and unprocessed samples are compared under the same chromatographic conditions.

\section{LC-MS chromatographic profiling of Croton tiglium seeds}

The test solution was prepared by dissolving ethanol extracts of Croton tiglium seeds before $(20.8 \mathrm{mg})$ and after $(20.7 \mathrm{mg})$ shodhana process in HPLC grade methanol up to $1.0 \mathrm{ml}$ volume. It is filtered and sent for LC-MS analysis. The ethanol extracts of the Croton tiglium seeds before and after the shodhana process, the processed and unprocessed samples are compared under the same chromatographic conditions. 


\section{Estimation of Crotonoside}

Ten $\mu$ each of the extract test solutions was injected into the HPLC system. The chromatogram was recorded, the area of peak of test solution was determined corresponding to that of crotonoside, described above from the calibration curve.

\section{Results and Discussion \\ Preliminary analysis}

Preliminary phytochemicals screening results showed the presence or absence of certain phytochemicals in the Croton tiglium seeds. The comparative preliminary phytochemicals screening results of Croton tiglium seeds before and after the shodhana process are tabulated in Table 1 .

Table 1: Preliminary phytochemicals screening of Croton tiglium seeds before and after shodhana process

\begin{tabular}{|c|c|c|c|}
\hline \multirow{2}{*}{ S. No. } & Parameters & $\begin{array}{c}\text { Croton tiglium } \\
\text { Before } \\
\text { Shodhana }\end{array}$ & $\begin{array}{c}\text { After } \\
\text { Shodhana }\end{array}$ \\
\hline 1 & Alkaloids & + & + \\
\hline 2 & Coumarins & + & + \\
\hline 3 & Flavanoids & + & + \\
\hline 4 & Glycosides & + & + \\
\hline 5 & Phenols & + & + \\
\hline 6 & Proteins & + & + \\
\hline 7 & Saponins & + & + \\
\hline 8 & Steroids & + & + \\
\hline 9 & Sugar & - & - \\
\hline 10 & Tannins & + & ++ \\
\hline
\end{tabular}

Physico-chemical analysis

The comparative analysis results of physicochemical parameters for Croton tiglium seeds samples before and after shodhana processes are tabulated in Table 2. The results of all parameters are complying the Ayurvedic Pharmacopeia of India (API) standards.

Table 2: Physicochemical parameters of Croton tiglium seeds before and after shodhana process

\begin{tabular}{|c|c|c|c|}
\hline S. No. & Parameters & \multicolumn{2}{|c|}{ Croton tiglium } \\
\hline 1 & $\begin{array}{c}\text { Before } \\
\text { Shodhana }\end{array}$ & $\begin{array}{c}\text { After } \\
\text { Shodhana }\end{array}$ \\
\hline 2 & $\mathrm{pH}(10 \%$ w/v aqueous solution) & 5.66 & 3.24 \\
\hline 3 & $\begin{array}{c}\text { Loss on drying at } 105^{\circ} \mathrm{C} \\
(\% \mathrm{w} / \mathrm{w})\end{array}$ & 6.82 & 9.49 \\
\hline 4 & $\begin{array}{c}\text { Water soluble extractive } \\
(\% \mathrm{w} / \mathrm{w})\end{array}$ & 8.20 & 12.41 \\
\hline 5 & $\begin{array}{c}\text { Alcohol soluble extractive } \\
(\% \mathrm{w} / \mathrm{w})\end{array}$ & 39.52 & 45.77 \\
\hline 6 & $\begin{array}{c}\text { Ash content } \\
(\% \mathrm{w} / \mathrm{w})\end{array}$ & 2.68 & 2.58 \\
\hline & $\begin{array}{c}\text { Acid-insoluble ash } \\
(\% \mathrm{w} / \mathrm{w})\end{array}$ & 0.21 & 0.20 \\
\hline
\end{tabular}

The percentage of water-soluble extractive, alcohol soluble extractive, and loss on drying at $105^{\circ} \mathrm{C}$ were found to increase ash content, acid-insoluble ash, and $\mathrm{pH}$ value reduced in the shodhit (processed) as compare to ashodhit (unprocessed) Croton tiglium seeds sample.

\section{Extraction procedure}

The total Ethanol and Chloroform extracts of Croton tiglium seeds are extracted using the Soxhlet method, and the obtained values are tabulated and given below:

Table 3: Extractive values of Croton tiglium seeds before and after shodhana process

\begin{tabular}{|c|c|c|c|c|c|c|c|}
\hline \multirow{2}{*}{$\begin{array}{l}\text { S. } \\
\text { No. }\end{array}$} & \multirow{2}{*}{$\begin{array}{l}\text { Name of } \\
\text { extracts }\end{array}$} & \multicolumn{2}{|c|}{$\begin{array}{l}\text { Weight of } \\
\text { sample (g) }\end{array}$} & \multicolumn{2}{|c|}{$\begin{array}{l}\text { Weight of } \\
\text { extract (g) }\end{array}$} & \multicolumn{2}{|c|}{$\begin{array}{c}\text { Extractive value } \\
\%(w / w)\end{array}$} \\
\hline & & Before & f & offor & fte & & Aftor \\
\hline 1 & $\begin{aligned} \mathrm{Chl} \\
\mathrm{e}\end{aligned}$ & 10.0432 & & 4.4538 & 7.4086 & & \\
\hline 2 & & 10.0582 & 12.049 & 4.1999 & 7.2345 & 41.1212 & 00.0 \\
\hline
\end{tabular}

\section{Identification of Bio-marker by HPTLC:}

The calculated $R_{f}$ value details were given in Tables 4 to 6 . A band at $254 \mathrm{~nm}$ (Blue, $\mathrm{R}_{\mathrm{f}} 0.54$ ) corresponding crotonoside is visible in both the reference standard and test solution tracks of chloroform and ethanol extracts of Croton tiglium seeds before and after the shodhana process.

Table 4: $\mathbf{R}_{\mathbf{f}}$ values of Croton tiglium seeds chloroform \& ethanol extracts before and after shodhana process and Crotonoside reference standard at UV $254 \mathrm{~nm}$

\begin{tabular}{|c|c|c|c|c|c|c|}
\hline \multirow{2}{*}{ Tracks } & \multicolumn{3}{|c|}{ Before Shodhana } & \multicolumn{3}{|c|}{ After Shodhana } \\
\hline & Peak & $\mathbf{R}_{\mathbf{f}}$ & Color & Peak & $\mathbf{R}_{\mathbf{f}}$ & Color \\
\hline \multirow{3}{*}{$\begin{array}{c}\text { Track } 1 \text { \& } 5 \\
\text { Chloroform } \\
\text { extract }\end{array}$} & 1 & 0.02 & Green & - & - & - \\
\hline & 2 & 0.54 & Green & - & - & - \\
\hline & 3 & 0.82 & Green & 1 & 0.89 & Green \\
\hline \multirow{7}{*}{$\begin{array}{c}\text { Track } 2 \& 4 \\
\text { Ethanol } \\
\text { extract }\end{array}$} & 1 & 0.02 & Green & - & - & - \\
\hline & 2 & 0.15 & Green & - & - & - \\
\hline & 3 & 0.18 & Green & - & - & - \\
\hline & 4 & 0.54 & Green & - & - & - \\
\hline & 5 & 0.62 & Green & - & - & - \\
\hline & 6 & 0.77 & Green & - & - & - \\
\hline & 7 & 0.96 & Green & 1 & 0.89 & Green \\
\hline $\begin{array}{c}\text { Track-3 } \\
\text { Crotonoside } \\
\text { standard }\end{array}$ & 1 & 0.54 & Green & - & - & - \\
\hline
\end{tabular}

Table 5: $\mathbf{R}_{\mathrm{f}}$ values of Croton tiglium seeds chloroform \& ethanol extracts before and after shodhana process at $\mathrm{UV} 366 \mathrm{~nm}$

\begin{tabular}{|c|c|c|c|c|c|c|}
\hline \multirow{2}{*}{ Tracks } & \multicolumn{3}{|c|}{ Before shodhana } & \multicolumn{3}{|c|}{ After shodhana } \\
\hline & Peak & $\mathbf{R}_{\mathbf{f}}$ & Color & Peak & $\mathbf{R}_{\mathbf{f}}$ & Color \\
\hline \multirow{4}{*}{$\begin{array}{l}\text { Track 1 } \\
\text { \& } 5 \\
\text { Chlorof } \\
\text { orm } \\
\text { extract }\end{array}$} & 1 & 0.15 & $\begin{array}{l}\text { Pale } \\
\text { yellow }\end{array}$ & & - & - \\
\hline & 2 & 0.57 & Blue & 1 & .63 & Blue \\
\hline & - & 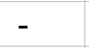 & - & 2 & 9 & lue \\
\hline & 3 & 0.85 & Blue & 3 & .89 & Blue \\
\hline \multirow{6}{*}{$\begin{array}{l}\text { Track } 2 \\
\& 4 \\
\text { Ethanol } \\
\text { extract }\end{array}$} & 1 & & & - & - & - \\
\hline & 2 & 0.59 & Blu & - & - & - \\
\hline & 3 & 0.62 & Blue & 1 & 0.63 & Blue \\
\hline & 4 & 0.69 & Blue & - & - & - \\
\hline & 5 & 0.78 & Blue & 2 & 0.79 & Blue \\
\hline & 6 & 0.96 & Blue & 3 & 0.89 & Blue \\
\hline
\end{tabular}


Ajay Kumar Meena et.al., Evaluation of the role of Shodhana (Purification) process in Croton tiglium seeds

Table 6: $\mathbf{R}_{\mathrm{f}}$ values of Croton tiglium seeds chloroform $\&$ ethanol extracts before and after shodhana process at $540 \mathrm{~nm}$

\begin{tabular}{|l|r|l|l|r|r|r|}
\hline \multirow{2}{*}{ Tracks } & \multicolumn{3}{|c|}{ Before shodhana } & \multicolumn{3}{c|}{ After shodhana } \\
\hline & Peak & $\mathbf{R}_{\mathbf{f}}$ & Color & Peak & \multicolumn{1}{c|}{$\mathbf{R}_{\mathbf{f}}$} & Color \\
\hline $\begin{array}{l}\text { Track 1 \& 5 } \\
\text { Chloroform } \\
\text { extract }\end{array}$ & 1 & 0.58 & Grey & 1 & 0.63 & Grey \\
\hline & 2 & 0.77 & Grey & 2 & 0.88 & Grey \\
\hline Track 2 \& 4 & 1 & 0.17 & Brown & - & - & - \\
\hline Ethanol & 2 & 0.56 & Brown & - & - & - \\
\hline extract & 3 & 0.60 & Grey & 1 & 0.63 & Grey \\
& 4 & 0.86 & Grey & 2 & 0.88 & Grey \\
\hline & 5 & 0.96 & Grey & - & - & - \\
\hline
\end{tabular}

Chemical profiling of Croton tiglium seeds

The details of the chromatographic profiling were given below:

\section{HPTLC chromatographic profiling of Croton tiglium} seeds

The Fingerprint profile was performed for the above-developed plate (Figure 1) used for identification. The $\mathrm{R}_{\mathrm{f}}$ values and fingerprint data were recorded by WIN CATS software. Details of HPTLC fingerprint profiling are given in Figures $2 \& 3$.
Figure 1: HPTLC fingerprint Profiling of Croton tiglium seeds chloroform \& ethanol extracts before and after shodhana process and Crotonoside reference standard

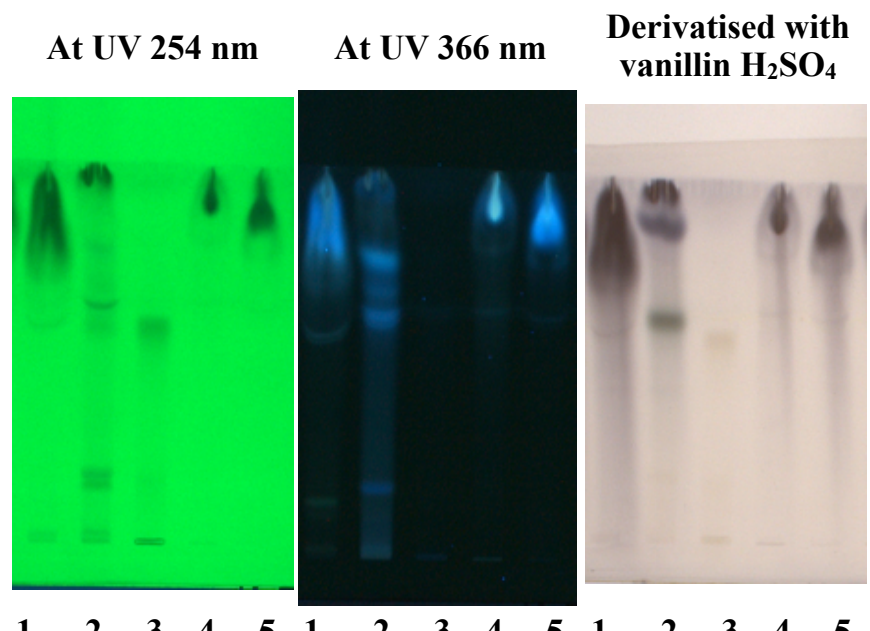

Chloroform extract: Track-1: Before Shodhana; Track-5: After Shodhana;

Reference standard: Track-3: Crotonoside Ethanol extract: Track-2: Before Shodhana; Track-4: After Shodhana

Figure 2: HPTLC fingerprint profile of Croton tiglium seeds chloroform \& ethanol extracts before and after shodhana process and Crotonoside reference standard at UV $254 \mathrm{~nm}$

Chloroform extract before Shodhana<smiles>[SiH3]</smiles>

m-

$\infty \infty-$

$\infty \times-$

(N)

$+\infty-$

m
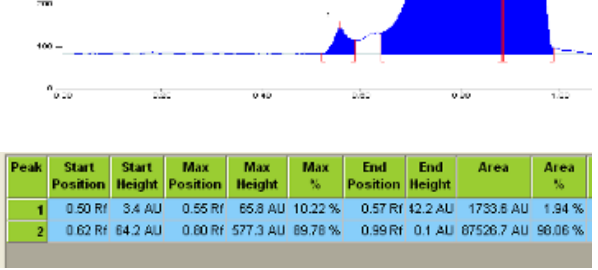

Ethanol extract after Shodhana
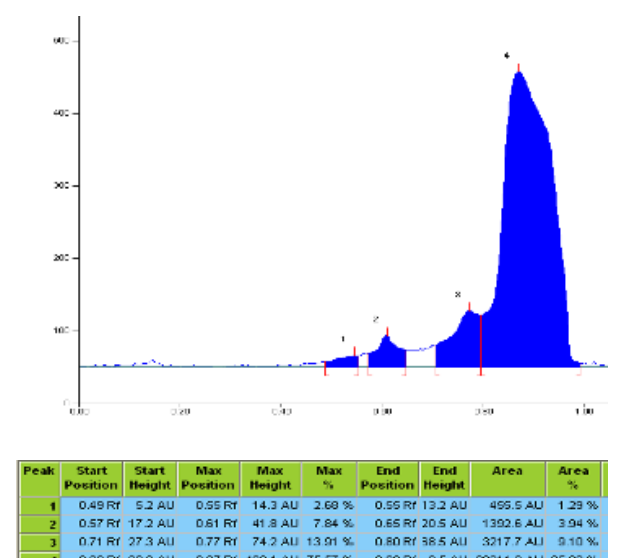

Ethanol extract before Shodhana
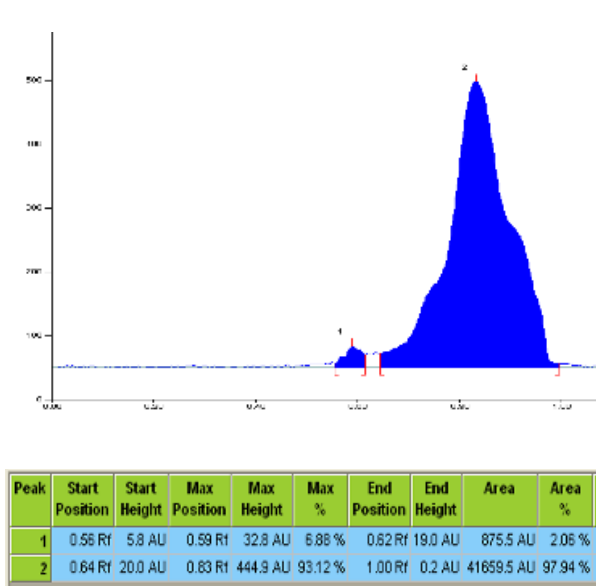

Crotonoside reference standard

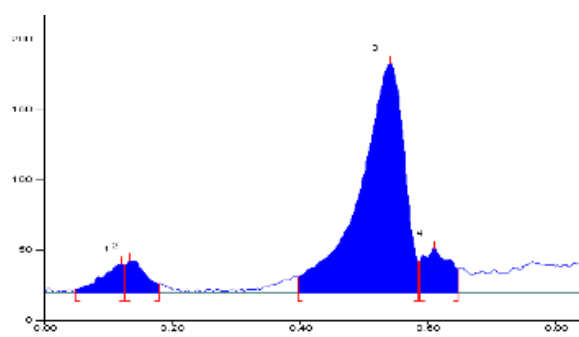

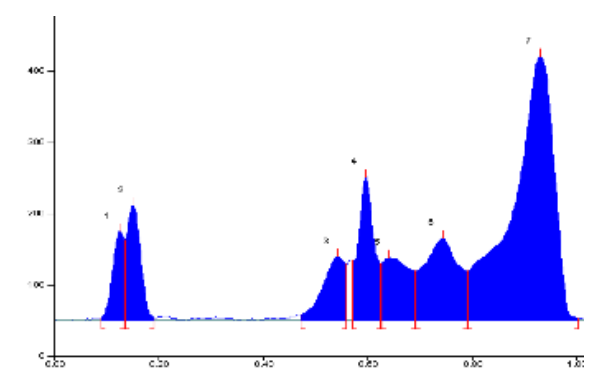

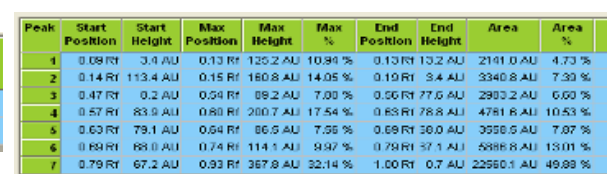


Figure 3: HPTLC fingerprint profile of Croton tiglium seeds chloroform \& ethanol extracts before and after shodhana process at UV 366nmHPLC chromatographic profiling of Croton tiglium seeds Chloroform extract before Shodhana

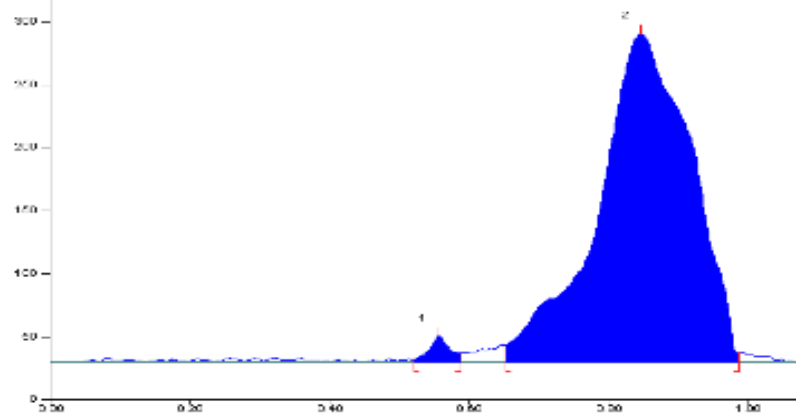

\begin{tabular}{|c|c|c|c|c|c|c|c|c|c|}
\hline tak & \begin{tabular}{|c|} 
Start \\
Position
\end{tabular} & \begin{tabular}{|c|}
$\begin{array}{c}\text { Start } \\
\text { Height }\end{array}$ \\
\end{tabular} & \begin{tabular}{|c|} 
Max \\
Position
\end{tabular} & $\begin{array}{c}\text { Max } \\
\text { Height }\end{array}$ & Max & \begin{tabular}{|c|} 
End \\
Position
\end{tabular} & \begin{tabular}{|c|} 
Endl \\
Height
\end{tabular} & Area & $\begin{array}{c}\text { Area } \\
\% 6\end{array}$ \\
\hline 1 & $0.51 \mathrm{Rf}$ & 5 AU & 1 & $22.1 \mathrm{suJ}$ & 9.5 & if & 8.3 & 62 & $2.11 \%$ \\
\hline 2 & $0.62 \mathrm{Rf}$ & $135 \mathrm{AU}$ & $0.78 \mathrm{Rt}$ & 2083 AJ & $90.42 \%$ & $1.00 R \mathrm{ft}$ & $0.4 \mathrm{AJ}$ & 29177.4 AJ & $97.99 \%$ \\
\hline
\end{tabular}

Chloroform extract after Shodhana

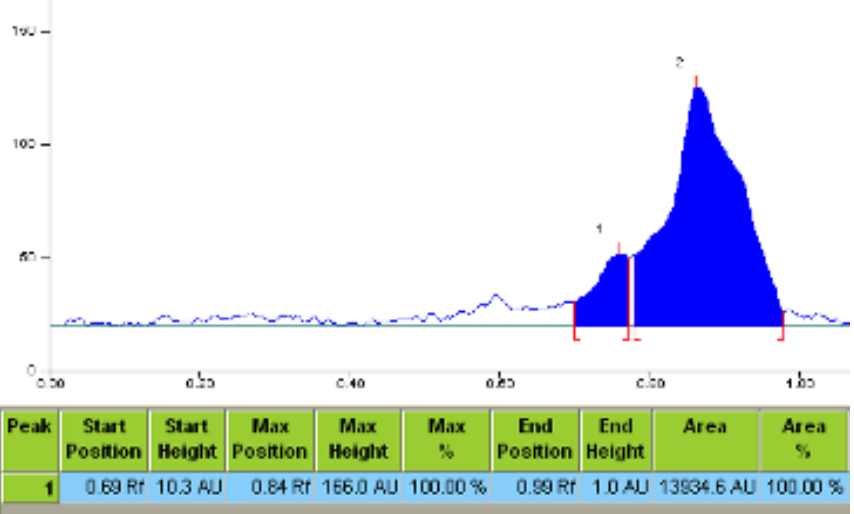

Ethanol extract before Shodhana
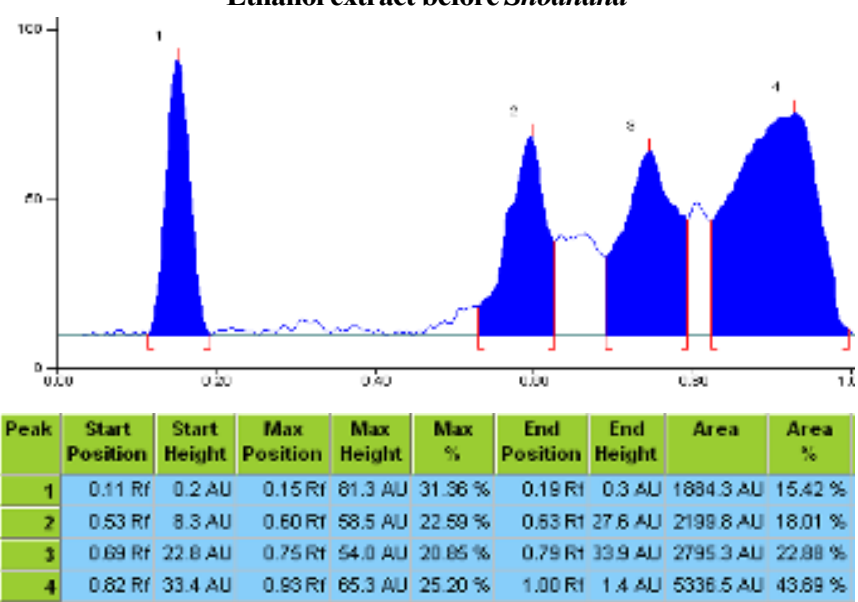

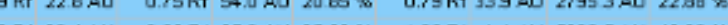
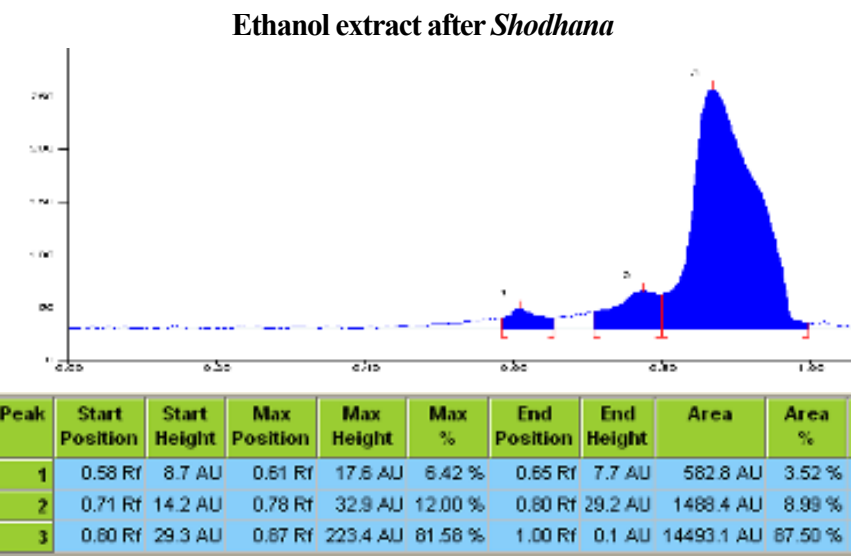

Figure 4: HPLC Profiling Chromatogram of Croton tiglium seeds chloroform extracts before and after shodhana process

Unprocessed sample

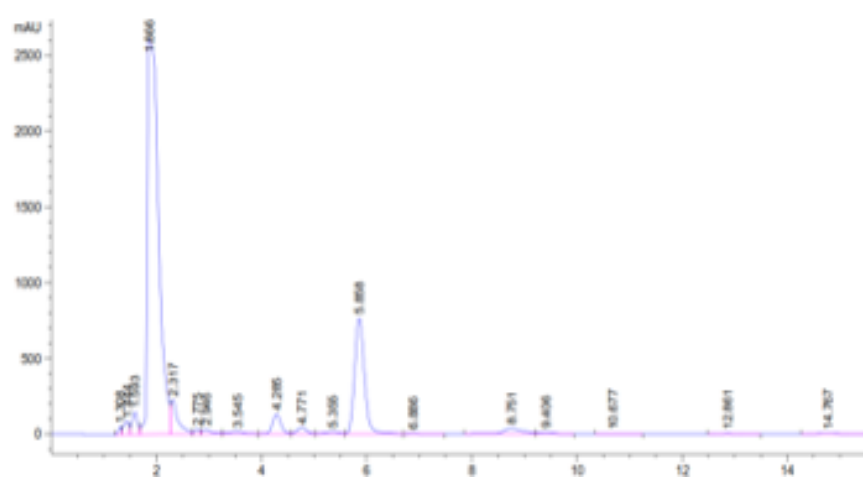

Processed sample

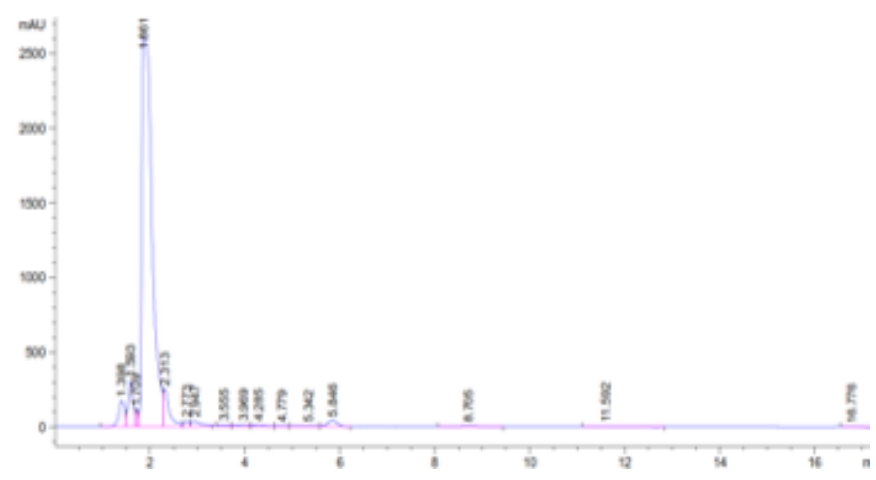

Figure 5: HPLC Profiling Chromatogram of Croton tiglium seeds ethanol extracts before and after shodhana process Unprocessed sample
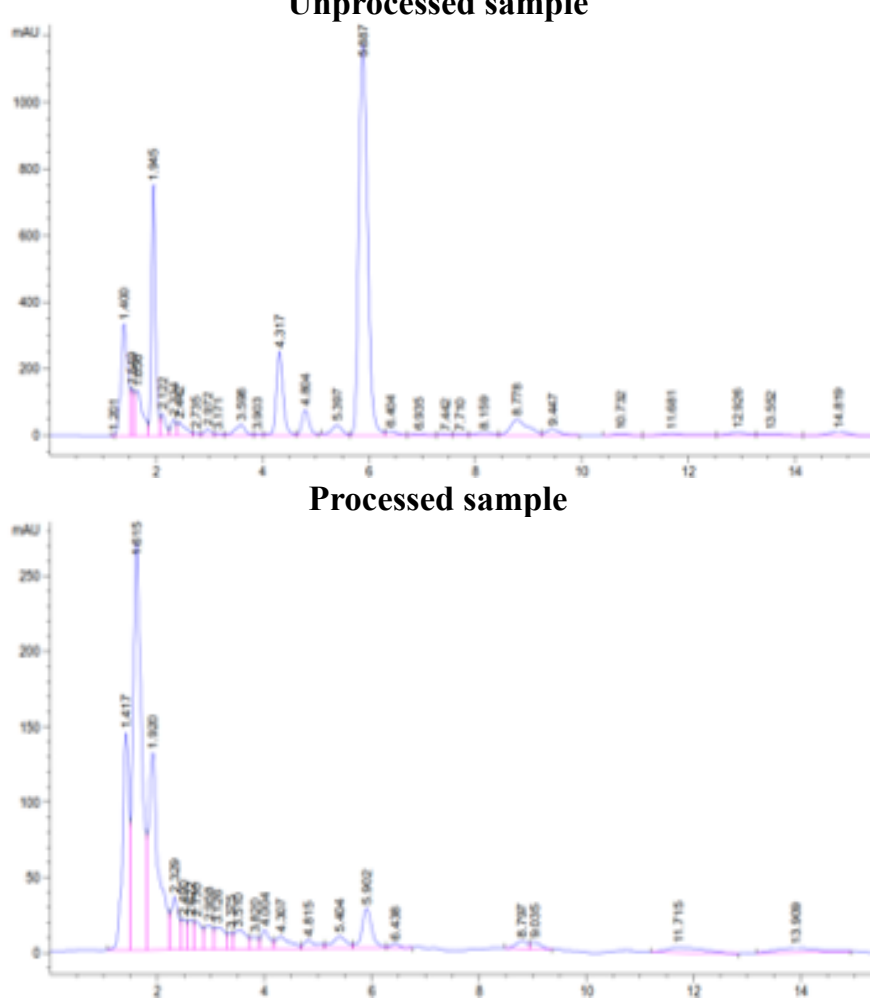
In comparing HPLC Chromatographic profiling of chloroform extracts, 18 peaks in unprocessed and 16 peaks in processed samples were detected. Twenty-nine peaks in processed and 22 peaks in unprocessed samples of each ethanol extract were detected. It is observed that the peak area of all peaks of processed samples was reduced as compared to unprocessed samples. The detailed peak identification and peak area results are shown in Figures $4 \& 5$ and Table $7 \& 8$. The remarkable changes have been observed in the chloroform and ethanol extracts HPLC profiling chromatograms of the Croton tiglium seeds before and after the shodhana process.

Table 7: HPLC peak details of Croton tiglium seeds chloroform extracts before and after shodhana process Unprocessed sample

\begin{tabular}{|c|c|c|c|c|c|c|c|}
\hline $\begin{array}{l}\text { Peak } \\
\text { No. }\end{array}$ & $\begin{array}{l}\text { Ret. Time } \\
\text { [min] }\end{array}$ & $\begin{array}{c}\text { Area } \\
{\left[\mathbf{m A U} \mathbf{A U}^{*} \mathbf{s}\right]}\end{array}$ & $\begin{array}{c}\text { Area } \\
\%\end{array}$ & $\begin{array}{c}\text { Peak } \\
\text { No. }\end{array}$ & $\begin{array}{l}\text { Ret. Time } \\
\text { [min] }\end{array}$ & $\begin{array}{c}\text { Area } \\
{\left[\mathbf{m A U} \mathbf{U}^{*} \mathbf{s}\right]}\end{array}$ & $\begin{array}{c}\text { Area } \\
\%\end{array}$ \\
\hline 1 & 1.308 & 168.35727 & 0.2924 & 1 & 1.398 & 1564.19421 & 3.1961 \\
\hline 2 & 1.444 & 602.68860 & 1.0466 & 2 & 1.593 & 2100.35010 & 4.2916 \\
\hline 3 & 1.593 & 1066.77148 & 1.8525 & 3 & 1.709 & 437.55542 & 0.8940 \\
\hline 4 & 1.866 & 38122.7 & 66.2013 & 4 & 1.861 & 40008.1 & 81.7473 \\
\hline 5 & 2.317 & 2230.60718 & 3.8735 & 5 & 2.313 & 2240.00977 & 4.5769 \\
\hline 6 & 2.775 & 244.66501 & 0.4249 & 6 & 2.773 & 291.11786 & 0.5948 \\
\hline 7 & 2.946 & 446.94354 & 0.7761 & 7 & 2.947 & 579.47070 & 1.1840 \\
\hline 8 & 3.545 & 407.13733 & 0.7070 & 8 & 3.555 & 180.02390 & 0.3678 \\
\hline 9 & 4.285 & 1525.03955 & 2.6483 & 9 & 3.969 & 210.16249 & 0.4294 \\
\hline 10 & 4.771 & 574.77069 & 0.9981 & 10 & 4.285 & 218.60977 & 0.4467 \\
\hline 11 & 5.355 & 331.66455 & 0.5759 & 11 & 4.779 & 55.14981 & 0.1127 \\
\hline 12 & 5.858 & 10185.4 & 17.6873 & 12 & 5.342 & 118.92033 & 0.2430 \\
\hline 13 & 6.886 & 88.24789 & 0.1532 & 13 & 5.846 & 557.70709 & 1.1395 \\
\hline 14 & 8.751 & 1003.82599 & 1.7432 & 14 & 8.705 & 223.64122 & 0.4570 \\
\hline 15 & 9.406 & 236.68639 & 0.4110 & 15 & 11.592 & 135.29448 & 0.2764 \\
\hline 16 & 10.677 & 30.00565 & 0.0521 & 16 & 16.776 & 20.86800 & 0.0426 \\
\hline 17 & 12.861 & 64.25497 & 0.1116 & - & - & - & - \\
\hline 18 & 14.767 & 256.25562 & 0.4450 & - & - & - & - \\
\hline Total & 57586 & 100.0000 & Total & 48941.2 & 100.0000 & & \\
\hline
\end{tabular}

Table 8: HPLC peak details of Croton tiglium seeds ethanol extracts before and after shodhana process Unprocessed sample

\begin{tabular}{|c|c|c|c|c|c|c|c|}
\hline & & & & & & & \\
\hline $\begin{array}{c}\text { Peak } \\
\text { No. }\end{array}$ & $\begin{array}{c}\text { Ret. Time } \\
\text { [min] }\end{array}$ & $\begin{array}{c}\text { Area } \\
{[\mathbf{m A U} \mathbf{A}]}\end{array}$ & $\begin{array}{c}\text { Area } \\
\%\end{array}$ & $\begin{array}{c}\text { Peak } \\
\text { No. }\end{array}$ & $\begin{array}{c}\text { Ret. Time } \\
\text { [min] }\end{array}$ & $\begin{array}{c}\text { Area } \\
{\left[\mathbf{m A U} \mathbf{U}^{*} \mathbf{s}\right]}\end{array}$ & $\begin{array}{c}\text { Area } \\
\%\end{array}$ \\
\hline 1 & 1.201 & 6.83685 & 0.0221 & 1 & 1.417 & 1212.14758 & 13.7826 \\
\hline 2 & 1.400 & 3057.50342 & 9.9052 & 2 & 1.615 & 3033.32080 & 34.4900 \\
\hline 3 & 1.549 & 592.55927 & 1.9197 & 3 & 1.920 & 1679.63477 & 19.0981 \\
\hline 4 & 1.656 & 1340.63806 & 4.3432 & 4 & 2.329 & 331.62753 & 3.7707 \\
\hline 5 & 1.945 & 3969.21338 & 12.8588 & 5 & 2.490 & 139.77296 & 1.5893 \\
\hline 6 & 2.122 & 438.00662 & 1.4190 & 6 & 2.622 & 138.78291 & 1.5780 \\
\hline 7 & 2.334 & 310.46707 & 1.0058 & 7 & 2.730 & 182.34494 & 2.0733 \\
\hline 8 & 2.442 & 441.22461 & 1.4294 & 8 & 2.958 & 175.94273 & 2.0005 \\
\hline 9 & 2.235 & 80.55502 & 0.2610 & 9 & 3.126 & 191.78812 & 2.1807 \\
\hline 10 & 2.972 & 189.42357 & 0.6137 & 10 & 3.375 & 71.05652 & 0.8079 \\
\hline 11 & 3.171 & 64.68430 & 0.2096 & 11 & 3.510 & 191.54918 & 2.1780 \\
\hline 12 & 3.598 & 468.20312 & 1.5168 & 12 & 3.820 & 82.56252 & 0.9388 \\
\hline 13 & 3.903 & 45.03476 & 0.1459 & 13 & 4.004 & 137.13824 & 1.5593 \\
\hline 14 & 4.317 & 2235.47900 & 7.2421 & 14 & 4.307 & 149.76952 & 1.7029 \\
\hline 15 & 4.804 & 750.93158 & 2.4327 & 15 & 4.815 & 91.74092 & 1.0431 \\
\hline 16 & 5.397 & 465.05450 & 1.5066 & 16 & 5.404 & 143.11061 & 1.6272 \\
\hline 17 & 5.887 & 13258.5 & 42.9525 & 17 & 5.902 & 339.80380 & 3.8637 \\
\hline 18 & 6.404 & 209.98225 & 0.6803 & 18 & 6.438 & 32.85186 & 0.3735 \\
\hline 19 & 6.935 & 99.92309 & 0.3237 & 19 & 8.797 & 74.11476 & 0.8427 \\
\hline 20 & 7.442 & 56.83461 & 0.1841 & 20 & 9.035 & 65.72379 & 0.7473 \\
\hline 21 & 7.710 & 57.35696 & 0.1858 & 21 & 11.715 & 159.79659 & 1.8169 \\
\hline 22 & 8.159 & 195.26073 & 0.6326 & 22 & 13.909 & 170.20543 & 1.9353 \\
\hline
\end{tabular}




\begin{tabular}{|c|c|c|c|c|c|c|}
\hline 23 & 8.778 & 1106.25854 & 3.5839 & - & - & - \\
\hline 24 & 9.447 & 326.18021 & 1.0567 & - & - & - \\
\hline 25 & 10.732 & 84.68050 & 0.2743 & - & - & - \\
\hline 26 & 11.681 & 225.43211 & 0.7303 & - & - & - \\
\hline 27 & 12.926 & 246.15454 & 0.7974 & - & - & - \\
\hline 28 & 13.552 & 169.09544 & 0.5478 & - & - & - \\
\hline 29 & 14.819 & 376.28485 & 1.2190 & - & - & - \\
\hline Total & $\mathbf{3 0 8 6 7 . 7}$ & $\mathbf{1 0 0 . 0 0 0 0}$ & Total & $\mathbf{8 7 9 4 . 7 8 6 0 9}$ & $\mathbf{1 0 0 . 0 0 0 0}$ & - \\
\hline
\end{tabular}

\section{GC-MS chromatographic profiling of Croton tiglium} seeds

GC-MS analysis of hexane extracts of Croton tiglium seeds shows the presence of 6 peaks in the unprocessed sample and eight peaks in the processed sample. The detailed peak identification is shown in Figure 6, and retention time, peak area, area percentage, compound name, and molecular weight are given in Table 9. The chloroform extracts of Croton tiglium

Figure 6: GC-MS Profiling Chromatogram of Croton tiglium seeds hexane extracts before and after shodhana process

Unprocessed sample

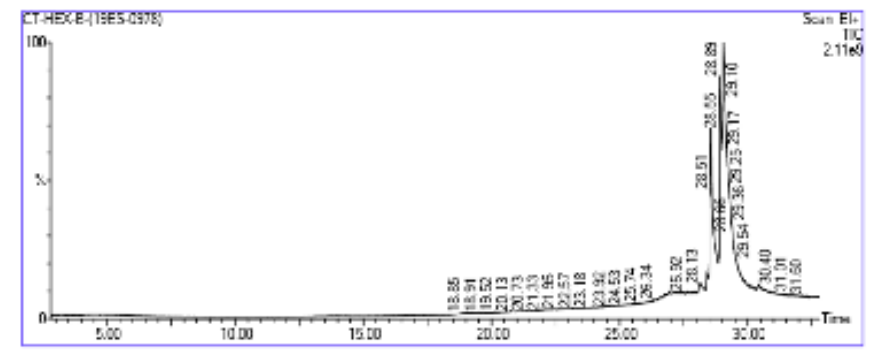

Processed sample

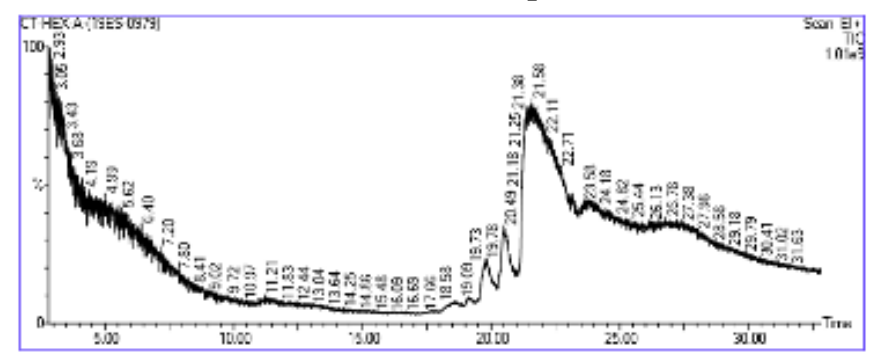

seeds show ten peaks in the unprocessed sample and 12 peaks in the processed sample. The detailed peak identification is shown in Figure 7, and retention time, peak area, area percentage, compound name, and molecular weight are given in Table 10. The remarkable changes have been observed in the hexane and chloroform extracts, GC-MS profiling chromatograms of the Croton tiglium seeds before and after the shodhana process.
Figure 7: GC-MS Profiling Chromatogram of Croton tiglium seeds chloroform extracts before and after shodhana process

Unprocessed sample

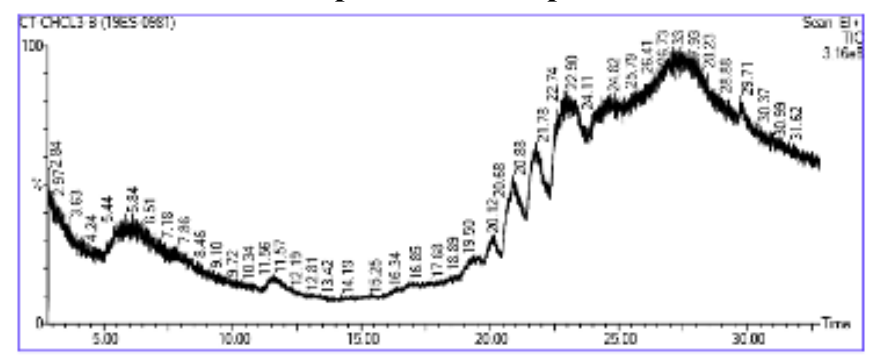

Processed sample

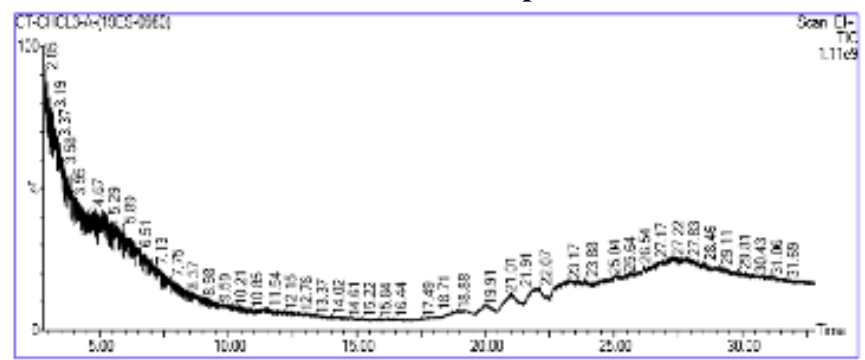

Table 9: GC-MS Peak details of Croton tiglium seeds hexane extracts before and after shodhana process Unprocessed sample

\begin{tabular}{|c|c|c|c|c|c|}
\hline Peak & RT & Area & Area \% & Name of the compound & Molecular weight \\
\hline 1 & 28.099 & $1,51,89,568$ & 1.583 & $\begin{array}{l}\text { 2,4,4-Trimethyl-3-hydroxymethyl-5a-(3-methyl -but-2- } \\
\text { enyl)-Cyclohexene }\end{array}$ & 222 \\
\hline 2 & 28.399 & $1,31,91,537$ & 1.375 & $\begin{array}{l}\text { 1,5-Cyclodecadiene, 1,5-dimethyl-8- (1-methylethenyl)-,[S- } \\
\text { (Z,E)]- }\end{array}$ & 204 \\
\hline 3 & 28.549 & $19,86,00,032$ & 20.698 & $\begin{array}{c}\text { 4,4,6A,6B,8A,11,11,14B-octamethyl-1,4,4A,5, 6,6A, } \\
\text { 6B,7,8,8A,9,10,11,12, 12A, 14,14A, }\end{array}$ & 424 \\
\hline 4 & 28.909 & $18,89,37,664$ & 19.691 & \multirow{2}{*}{$\begin{array}{l}\text { 2R-Acetoxymethyl-1,3,3-trimethyl-4t-(3-methyl -2-buten-1- } \\
\text { yl)-1t-cyclohexane }\end{array}$} & 282 \\
\hline 5 & 29.039 & $52,89,20,512$ & 55.123 & & 282 \\
\hline 6 & 30.430 & $1,46,84,390$ & 1.530 & $\begin{array}{l}\text { 2,4,4-Trimethyl-3-hydroxymethyl-5a- (3-methyl-but-2- } \\
\text { enyl)-cyclohexene }\end{array}$ & 222 \\
\hline
\end{tabular}


Processed sample

\begin{tabular}{|c|c|c|c|c|c|}
\hline Peak & RT & Area & Area $\%$ & Name of the compound & Molecular weight \\
\hline 1 & 19.806 & $5,87,19,072$ & 2.480 & Tetradecanoic acid & 228 \\
\hline 2 & 20.491 & $10,25,38,400$ & 4.330 & N-Hexadecanoic acid & 256 \\
\hline 3 & 21.581 & $1,02,92,25,664$ & 43.462 & 6-Heptadecyne, 1-chloro- & 270 \\
\hline 4 & 23.137 & $9,26,23,368$ & 3.911 & 9,12 Octadecadienoyl chloride, (Z,Z) & 298 \\
\hline 5 & 23.767 & $56,00,32,768$ & 23.649 & 17-Octadecynoic Acid & 308 \\
\hline 6 & 25.828 & $5,47,76,220$ & 2.313 & Z,Z-6,13-Octadecadien-1-Ol acetate & 152 \\
\hline 7 & 26.208 & $5,00,04,384$ & 2.112 & 2-Methyl-6-methylene-octa-1,7-dien-3-ol & 308 \\
\hline 8 & 26.783 & $42,02,07,360$ & 17.744 & Z,Z-6,13-octadecadien-1-ol acetate & P \\
\hline
\end{tabular}

Table 10: GC-MS Peak details of Croton tiglium seeds chloroform extracts before and after shodhana process Unprocessed sample

\begin{tabular}{|c|c|c|c|c|c|}
\hline Peak & RT & Area & Area \% & Name of the compound & Molecular weight \\
\hline 1 & 20.676 & $48,76,207.0$ & 0.985 & Tetradecanoic acid & 228 \\
\hline 2 & 20.881 & $48,84,822.5$ & 0.987 & Octadecanoic acid & 284 \\
\hline 3 & 21.776 & $2,56,05,386.0$ & 5.173 & Eicosanoic acid & 312 \\
\hline 4 & 22.847 & $3,33,04,378.0$ & 6.729 & Oleic acid & 282 \\
\hline 5 & 22.987 & $6,61,82,716.0$ & 13.372 & 9-Octadecenal & 266 \\
\hline 6 & 24.337 & $3,68,69,356.0$ & 7.449 & \multirow{2}{*}{ 1-Hexyl-2-nitrocyclohexane } & 213 \\
\hline 7 & 24.823 & $3,46,45,632.0$ & 7.000 & & 213 \\
\hline 8 & 27.118 & $19,53,49,632.0$ & 39.469 & 4-Pentadecyne, 15-chloro- & 242 \\
\hline 9 & 27.519 & $8,80,95,824.0$ & 17.799 & 2,6-Pyrazinediamine & 110 \\
\hline 10 & 29.710 & $51,30,300.5$ & 1.037 & Pregnan-3,11-diol-20-one & 334 \\
\hline \multicolumn{6}{|c|}{ Processed sample } \\
\hline Peak & RT & Area & Area \% & Name of the compound & Molecular weight \\
\hline 1 & 20.946 & $12,95,488.9$ & 3.000 & Tetradecanoic acid & 228 \\
\hline 2 & 24.823 & $12,68,241.5$ & 2.936 & Oleic acid & 282 \\
\hline 3 & 25.073 & $31,64,184.2$ & 7.326 & 2-Piperidinone, N-[4-bromo-N-butyl]- & 233 \\
\hline 4 & 26.168 & $25,08,068.8$ & 5.807 & 2,7-Octadiene-1,6-diol, 2,6-dimethyl-, (Z)- & 170 \\
\hline 5 & 26.678 & $18,02,641.9$ & 4.174 & Oleic acid & 282 \\
\hline 6 & 26.893 & $41,10,459.0$ & 9.517 & D-mannitol, 1-O-(22-hydroxydocosyl)- & 506 \\
\hline 7 & 27.299 & $62,32,076.0$ & 14.429 & $\begin{array}{l}\text { Estran-3-one, 17-(acetyloxy)-2-methyl-, } \\
\text { (2.alpha.,5.alpha.,17.beta.)- }\end{array}$ & 332 \\
\hline 8 & 27.459 & $14,46,128.2$ & 3.348 & $\begin{array}{l}\text { 2-isopropyl-5-methylcyclohexyl 3-(1-(4- } \\
\text { chlorophenyl)-3-oxobutyl)-coumarin }\end{array}$ & 524 \\
\hline 9 & 27.834 & $1,65,16,810.0$ & 38.242 & Di-N-decylsulfone & 346 \\
\hline 10 & 28.464 & $12,07,268.4$ & 2.795 & 2,6-Lutidine 3,5-dichloro-4-dodecylthio- & 375 \\
\hline 11 & 28.599 & $22,21,505.5$ & 5.144 & Cholesta-8,24-dien-3-ol, 4-methyl-, (3.beta.,4.alpha.)- & 398 \\
\hline 12 & 28.909 & $14,17,292.9$ & 3.282 & Pseduosarsasapogenin-5,20-Dien methyl ether & 428 \\
\hline
\end{tabular}

LC-MS chromatographic profiling of Croton tiglium seeds

LC-MS Chromatographic profiling of ethanol extracts of Croton tiglium seeds shows 19 peaks in the unprocessed sample and 17 peaks in the processed sample. The detailed peak identification is shown in
Figure 8 , and retention time, peak area, and area percentage are given in Table 11. The remarkable changes have been observed in the ethanol extracts LCMS profiling chromatograms of the Croton tiglium seeds before and after the shodhana process. 
Figure 8: LC-MS Chromatogram of Croton tiglium seeds ethanol extracts before and after shodhana process Unprocessed sample

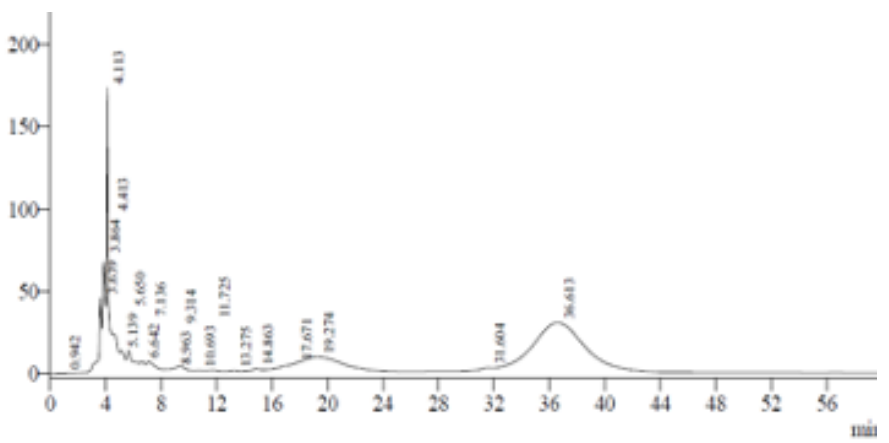

Processed sample

Table 11: LC-MS Peak details of Croton tiglium seeds ethanol extracts before and after shodhana process

Unprocessed sample

Processed sample

\begin{tabular}{|c|c|c|c|c|c|c|c|}
\hline Peak & Ret. Time & Area & Area \% & Peak & Ret. Time & Area & Area $\%$ \\
\hline 1 & 0.942 & 2010 & 0.019 & 1 & 3.329 & 2448 & 0.124 \\
\hline 2 & 3.639 & 170426 & 1.647 & 2 & 3.834 & 887 & 0.045 \\
\hline 3 & 3.864 & 279746 & 2.704 & 3 & 4.113 & 71707 & 3.646 \\
\hline 4 & 4.113 & 664843 & 6.425 & 4 & 4.979 & 21523 & 1.094 \\
\hline 5 & 4.413 & 64347 & 0.622 & 5 & 5.077 & 16188 & 0.823 \\
\hline 6 & 5.139 & 36854 & 0.356 & 6 & 6.362 & 29261 & 1.488 \\
\hline 7 & 5.650 & 75609 & 0.731 & 7 & 7.358 & 5694 & 0.289 \\
\hline 8 & 6.642 & 15949 & 0.154 & 8 & 8.818 & 16927 & 0.861 \\
\hline 9 & 7.136 & 70283 & 0.679 & 9 & 9.699 & 4266 & 0.217 \\
\hline 10 & 8.963 & 8341 & 0.081 & 10 & 10.204 & 0 & 0.000 \\
\hline 11 & 9.314 & 91690 & 0.886 & 11 & 10.620 & 8833 & 0.449 \\
\hline 12 & 10.693 & 2037 & 0.020 & 12 & 13.137 & 1684 & 0.086 \\
\hline 13 & 11.725 & 8596 & 0.083 & 13 & 14.713 & 42841 & 2.178 \\
\hline 14 & 13.275 & 7857 & 0.076 & 14 & 16.753 & 10959 & 0.557 \\
\hline 15 & 14.863 & 26000 & 0.251 & 15 & 19.552 & 271312 & 13.795 \\
\hline 16 & 17.671 & 186732 & 1.805 & 16 & 36.910 & 1437398 & 73.085 \\
\hline 17 & 19.274 & 1562917 & 15.104 & 17 & 45.237 & 24822 & 1.262 \\
\hline 18 & 31.604 & 20352 & 0.197 & - & - & - & - \\
\hline 19 & 36.613 & 7052972 & 68.161 & - & - & - & - \\
\hline Total & 10347561 & 100.000 & Total & 1966749 & 100.000 & & \\
\hline
\end{tabular}

Quantitative estimation of Crotonoside in Croton tiglium seeds by HPLC

Standard calibration curve for Crotonoside

Figure 9.

The calibration curve was established for peak area Vs. the concentration of Crotonoside applied is shown in

Figure 9: HPLC Chromatogram of Crotonoside standard and calibration curve
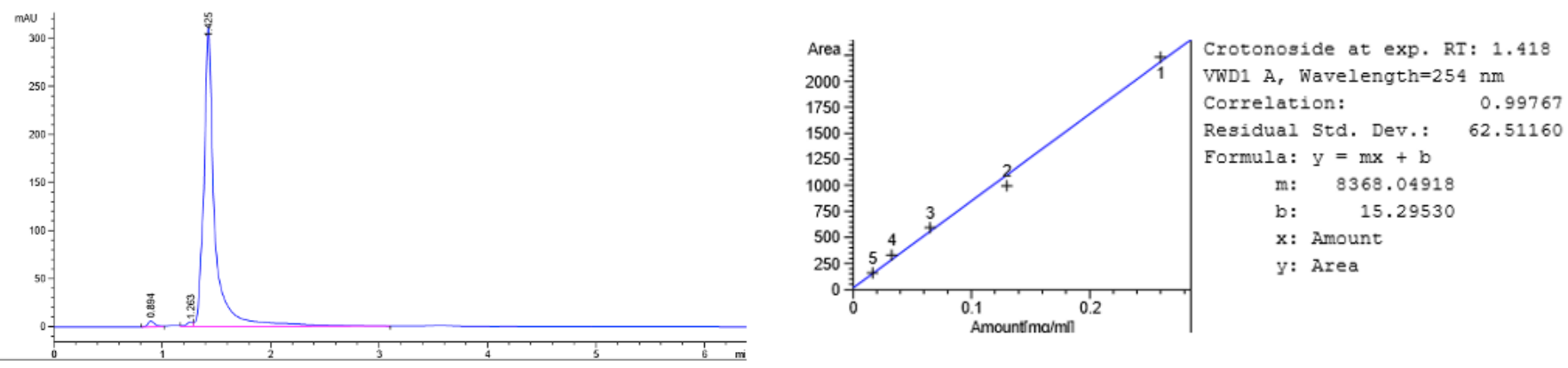

\section{Estimation of Crotonoside}

The calculated amount of crotonoside present in the residues extracted in ethanol and chloroform for each test sample obtained from before and after shodhana process samples of Croton tiglium seeds is given in Figure 10 and Table 12.

The results obtained from HPLC analysis revealed that the depletion in the level of crotonoside in ethanol and chloroform extracts after the shodhana process of Croton tiglium seeds compared to unprocessed seeds. HPLC analysis showed that the percentage of crotonoside was reduced by $2.43 \%$ in chloroform extract and $21.26 \%$ in ethanol extract after the shodhana process. 
Figure 10: Estimation of Crotonoside in the of Croton tiglium seeds chloroform and ethanol extracts of before and after shodhana process

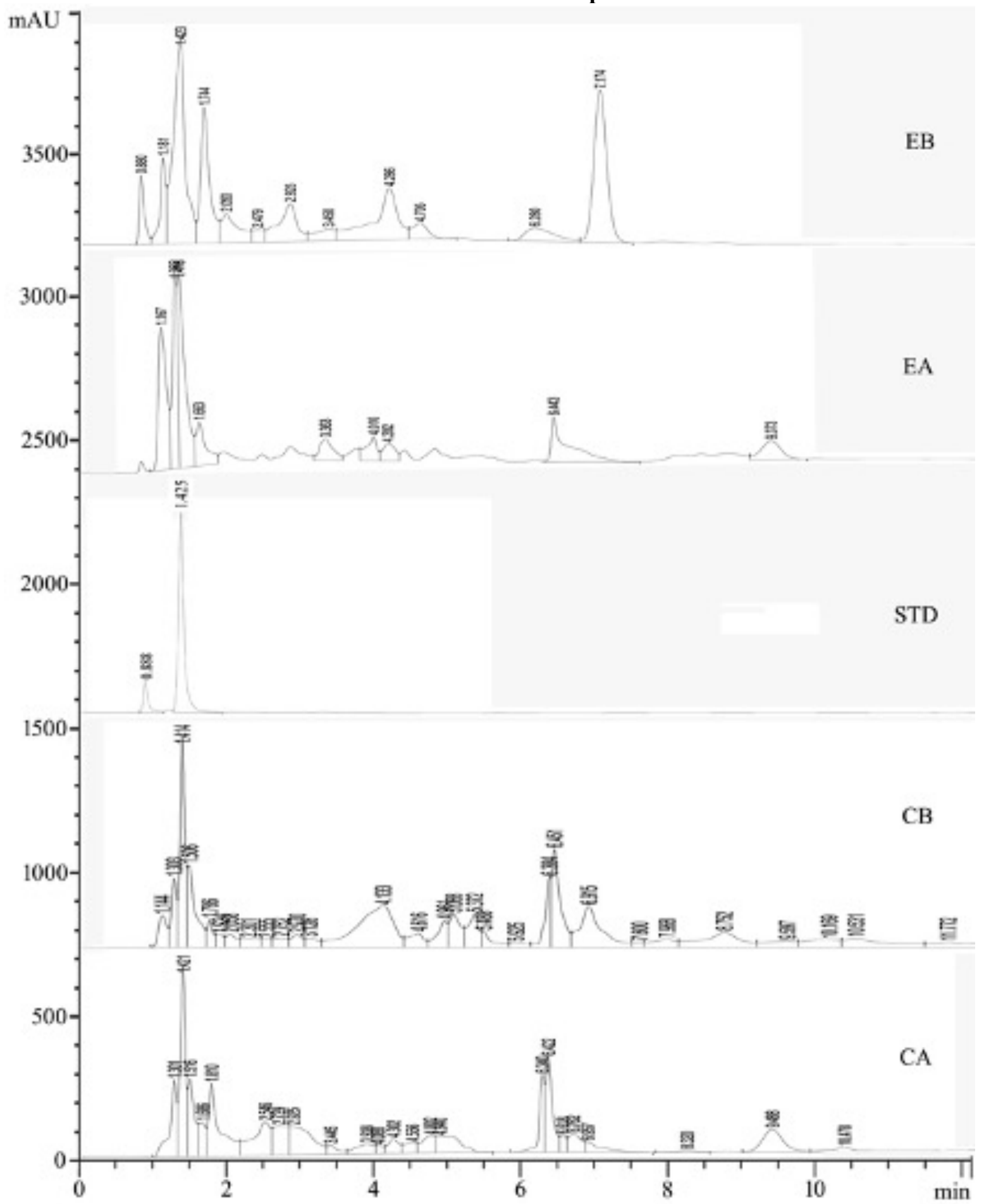

[EB-Ethanol before shodhana extract; EA- Ethanol after shodhana extract; STD- Crotonoside Standard; CBChloroform before shodhana extract; CA- Chloroform after shodhana extract]

Table 12: Estimation of Crotonoside in the of Croton tiglium chloroform and ethanol extracts of before and after shodhana process

\begin{tabular}{|c|c|c|c|c|c|c|}
\hline \multirow{3}{*}{ S. No. } & \multirow{3}{*}{ Name of extracts } & \multicolumn{5}{|c|}{ Crotonoside $(\% \mathrm{w} / \mathrm{w})$} \\
\hline & & \multicolumn{2}{|c|}{ Before Shodhana } & \multicolumn{2}{|c|}{ After Shodhana } & \multirow[t]{2}{*}{ Percentage reduced } \\
\hline & & Results & Mean & Results & Mean & \\
\hline \multirow{3}{*}{1} & \multirow{3}{*}{ Chloroform extract } & 0.0623 & \multirow{3}{*}{0.0623} & 0.0606 & \multirow{3}{*}{0.0608} & \multirow{3}{*}{$2.43 \%$} \\
\hline & & 0.0623 & & 0.0610 & & \\
\hline & & 0.0624 & & 0.0608 & & \\
\hline \multirow{3}{*}{2} & \multirow{3}{*}{ Ethanol extract } & 0.0816 & \multirow{3}{*}{0.0875} & 0.0694 & \multirow{3}{*}{0.0689} & \multirow{3}{*}{$21.26 \%$} \\
\hline & & 0.0943 & & 0.0690 & & \\
\hline & & 0.0866 & & 0.0684 & & \\
\hline
\end{tabular}

*Percentage of results was given from the means of triplicates for both before and after shodhana samples of optimized two solvent extracts of ethanol and chloroform. 


\section{Conclusion}

In this study, an attempt has been made to purify the Croton tiglium seeds by the classical Ayurvedic shodhana process. Preliminary phytochemical screening and physicochemical parameters were determined before and after the shodhana process of Croton tiglium seeds sample.

The percentage of water-soluble extractive, alcohol soluble extractive, and loss on drying at $105^{\circ} \mathrm{C}$ were found to increase ash content, acid-insoluble ash, and $\mathrm{pH}$ value reduced in the shodhit (processed) Croton tiglium seeds as compare to ashodhit (unprocessed) sample. The remarkable changes have been observed in different physicochemical parameters, HPTLC, HPLC, GC-MS, and LC-MS chromatographic profiling before and after the shodhana process of Croton tiglium seeds. In the HPLC chromatographic profiling, the peak area of all processed samples peak area was reduced compared to unprocessed samples.

The results obtained from HPLC analysis revealed that the depletion in the level of crotonoside in ethanol and chloroform extracts after the shodhana process of Croton tiglium seeds compared to unprocessed seeds. HPLC analysis showed that the percentage of crotonoside was reduced $2.43 \%$ in chloroform extract and $21.26 \%$ in ethanol extract after the shodhana process, respectively.

\section{Competing interests}

The authors declare that they have no competing interests.

\section{Acknowledgments}

The authors are very grateful to the DirectorGeneral, CCRAS, Ministry of AYUSH, New Delhi, for providing encouragement and facilities for carrying out this work.

\section{References}

1. Usman, M.R.M.; Salgar, S.D.; Nagpal, N.; Shaikh, M.Z. Poisonous Herbal Plants: NA; Educreation Publishing; New Delhi, 2016..

2. Pillai N. R., Gastro-intestinal effects of Croton tiglium in Experimental Animals. Ancient Science of Life. 1999; (3\&4): 205-209.

3. Nadkarni K.M., The Indian Materia Medica. Vol. 1, Popular Prakashan, Bombay; 1996, 396-397p.

4. Qiu H. X., Flora of China. Science Press; Beijing, 1996. 133p.
5. Wangx, Lan M, Wu HP, Shi YQ, Lu J, Ding J, et al., Direct effect of croton oil on intestinal epithelial cells and colonic smooth muscle cells. World J. Gastroenterol. 2002; 8: 103-107.

6. Tsai J. C., Tsai S., Chang W. C., Effect of ethanol extracts of three Chinese medicinal plants with laxative properties on ion transport of the rat intestinal epithelia. Biol. Pharm. Bull. 2004; 27:162-5.

7. Morimura K., The role of special group article in ancient Chinese medical prescription. Hist. Sci. (Tokyo) 2003; 13: 1-12.

8. Pandey G., Dravya Guna Vijnana, Vol. I, Edi. 2nd, Published by Krishnadas Academy; Varanasi, 2002, Reprint 2004, 862p.

9. Anonymous, The Ayurvedic Pharmacopoeia of India, Part-I, Vol-II. Govt. of India, Ministry of Health of Family Welfare; New Delhi: 2004, 20, 61-62, 177-179, 181p.

10. Anonymous, The Ayurvedic Formulary of India, Part- I,II\& III, 2nd Edition, Published by Department of Ayurveda, Yoga \& Naturopathy, Unani, Siddha and Homoeopathy (AYUSH), Ministry of Health and Family welfare, Govt. of India; New Delhi, 2011.

11. Pal P. K., Nandi M. K., Singh N. K., Detoxification of Croton tiglium L. seeds by Ayurvedic process of Śdhana. Ancient Science of Life, 2014; 33(3): 155-159.

12. Kalani J. and Nikam V. Phytochemical and PhysicoChemical Analysis of Jayapala Beeja (Croton tiglium Linn.) with reference to different Shodhana Samskara. IJOOAR, 2016; 1(1): 1-28.

13. Meena, A. J., Singh, A., Sharma, K., Kumari, S., \& Rao, M. M., Physicochemical and preliminary phytochemical studies on the Rhizomes of Glycyrrhiza glabra Linn. International Journal of Pharmacy and Pharmaceutical Sciences, 2010; 2, 48-50.

14. Sujatha K., Revenasiddappa S. S., Sweyha S., Analytical study on shodhana of Jayapala, Int. J. Res. Ayurveda Pharm, 2013; 4(6): 405-408.

15. Acharya R., Shodhana: An Ayurvedic detoxification Technique and its Impact on certain Medicinal Plants. In: Kumar A., Padhi M. M., Srikanth N., Dhar B. P., Mangal A. K. (Eds): conservation, cultivation and exploration of therapeutic potential of Medicinal plants, 1st edition, Central council for Research in Ayurvedic Sciences, New Delhi; 2014, 427-450p. 